\title{
PRÁTICAS INTEGRATIVAS COMPLEMENTARES NA ATENÇÃO PRIMÁRIA À SAÚDE
}

\author{
Pollyane da Costa Matos ${ }^{1}$, Carolina Rodrigues Laverde', Priscila Gomes Martins', \\ Juliana Martins de Souza², Nunila Ferreira de Oliveira², Calíope Pilger²
}

\begin{abstract}
RESUMO: Objetivo: analisar o conhecimento e as percepções de enfermeiros que trabalham na Atenção Primária de um município do sudeste goiano sobre as Práticas Integrativas e Complementares. Método: estudo descritivo e exploratório com abordagem qualitativa, com enfermeiros que atuam há mais de 6 meses na Atenção Básica. Os dados foram coletados por entrevista semiestruturada, no ano de 2015 e submetidos à análise temática de conteúdo. Resultados: emergiram três categorias: I - Conhecimento das enfermeiras sobre as Práticas Integrativas e Complementares, dificuldade de conceituá-las e percepção como tratamento complementar; II - Desafios e dificuldades na implementação das Práticas Integrativas e Complementares na Atenção Primária à Saúde; III - As Práticas Integrativas e Complementares como uma ferramenta de autocuidado e promoção da qualidade de vida. Conclusão: Evidenciou-se a necessidade de capacitação na graduação e em educação permanente, para utilizar as práticas como recurso de cuidado.

DESCRITORES: Atenção primária à saúde; Atenção integral à saúde; Terapias complementares; Saúde holística; Enfermagem.

\section{COMPLEMENTARY AND INTEGRATIVE PRACTICES IN PRIMARY HEALTH CARE}

ABSTRACT: Objective: to analyze the knowledge and perceptions of nurses working in Primary Health Care in a municipality of Southeastern Goiás on Complementary and Integrative Practices. Method: a descriptive and exploratory study with a qualitative approach, with nurses who have been working for more than 6 months in Primary Health Care. Data was collected by semi-structured interviews in 2015 and submitted to thematic content analysis. Results: three categories emerged: I - Nurses' knowledge about Complementary and Integrative Health Practices, difficulty in conceptualizing these practices and perception as a complementary treatment; II - Challenges and difficulties in implementing Complementary and Integrative Health Practices in Primary Health Care; III - Complementary and Integrative Health Practices as a tool for self-care and promotion of quality of life. Conclusion: Training on the use of these practices as a resource of care is necessary at graduation and in continuing education.

DESCRIPTORS: Primary health care; Integral health care; Complementary therapies; Holistic health; Nursing.
\end{abstract}

\section{PRÁCTICAS INTEGRATIVAS COMPLEMENTARIAS EN LA ATENCIÓN PRIMARIA DE SALUD}

RESUMEN: Objetivo: Analizar el conocimiento y las percepciones de enfermeros actuantes en la Atención Primaria de un municipio del sudeste de Goiás sobre las Prácticas Integrativas y Complementarias. Método: Estudio descriptivo y exploratorio, con abordaje cualitativo, realizado con enfermeros con más de 6 meses de actuación en Atención Primaria. Datos recolectados mediante entrevista semiestructurada durante 2015, sometidos a análisis temático de contenido. Resultados: Surgieron tres categorías: I - Conocimiento de las enfermeras sobre las Prácticas Integrativas y Complementarias, dificultades para conceptuarlas y percepción como tratamiento complementario; II - Desafíos y dificultades en la implementación de las Prácticas Integrativas y Complementarias en la Atención Primaria de Salud; III - Prácticas Integrativas y Complementarias como herramienta para el autocuidado y la promoción de la calidad de vida. Conclusión: Se evidenció la necesidad de capacitación en el curso de grado y en educación continua para la utilización de las prácticas como recurso de cuidado.

DESCRIPTORES: Atención Primaria de Salud; Atención Integral de Salud; Terapias Complementarias; Salud Holística; Enfermería.

${ }^{1}$ Discente do curso de graduação em Enfermagem, Universidade Federal de Goiás. Catalão-GO-Brasil.

²Enfermeira. Doutora em Ciências. Docente do Curso de Enfermagem da Universidade Federal de Goiás. Catalão-GO-Brasil. 


\section{INTRODUÇÃO}

O modelo biomédico vigente, hegemônico, considera o corpo humano como uma máquina complexa composta por diferentes e minuciosas partes que se relacionam obedecendo apenas a princípios naturais. As Práticas Integrativas Complementares (PIC) se opõem a esse modelo convencional, tendo como enfoque o olhar holístico sobre o ser humano, ao considerá-lo como uma unidade de mente/corpo/ espírito e não apenas como uma soma de partes isoladas ${ }^{(1)}$.

As PIC constituem um grupo de terapias e produtos que não fazem parte dos tratamentos médicos tradicionais. Elas são nomeadas complementares quando usadas em paralelo com a medicina convencional, alternativas quando usadas no lugar da prática biomédica ou quando substituem uma determinada técnica do campo da medicina convencional, e integrativas quando baseadas em avaliações científicas de eficácia e segurança ${ }^{(1-2)}$.

Essas práticas buscam estimular os mecanismos naturais de prevenção de agravos e promoção da saúde por meio de tecnologias eficazes e seguras, com base na escuta acolhedora, no desenvolvimento do vínculo terapêutico e na integração do ser humano com o meio ambiente e a sociedade ${ }^{(3-4)}$.

Em 2006, o Ministério da Saúde implantou a Política Nacional de Práticas Integrativas e Complementares (PNPIC) no Sistema Único de Saúde (SUS), consolidada pelas Portarias n. 971 de 03/05/06 e n. 1.600 de 17/07/06, e que busca incorporar essas práticas na Atenção Primária em Saúde. Suas diretrizes têm como foco principal garantir a prevenção, a promoção e a recuperação da saúde dos indivíduos, através de cuidado humanizado e integral. Além disso, a adoção da PNPIC propõe o fortalecimento da participação popular na implementação das PIC, pois é por meio dessa participação que novas ideias serão disseminadas ${ }^{(4)}$.

Em 2017, o Ministério da Saúde publicou a Portaria n. 849/2017, de 27 de março, a qual incluiu outros tipos de PIC nos serviços de saúde pública, ampliando cada vez mais o acesso para a população à arteterapia, à ayurveda, à biodança, à dança circular, à meditação, à musicoterapia, à naturopatia, à osteopatia, à quiropraxia, à reflexoterapia, ao reiki, à shantala, à terapia comunitária integrativa e ao yoga $^{(5)}$.

Outro aspecto importante a se ressaltar sobre as PIC é que, além de seus diversos benefícios, elas geram redução de gastos devido ao baixo custo na sua implementação, aliando eficiência terapêutica maior ou igual aos outros tratamentos, com viabilidade econômica que é importante para o sistema público de saúde ${ }^{(6)}$.

A teoria de Rogers aceita que o ser humano é um todo unificado que possui uma integralidade individual, manifestando características que são mais do que a soma das partes, e que fortalecem e fundamentam seus princípios em integralidade, ressonância e felicidade, além de operar com conceitos de campos de energia como sistemas abertos em constante troca ${ }^{(3,7)}$.

Considerando o pensamento de Rogers e sua importância para embasar a prática de Enfermagem e, consequentemente, direcionar o objeto de trabalho do enfermeiro - o cuidado - junto às PIC, o objetivo deste estudo foi analisar o conhecimento e as percepções de enfermeiros que trabalham na Atenção Primária de um município do sudeste do estado de Goiás sobre as Práticas Integrativas e Complementares.

\section{- MÉTODO}

Trata-se de um estudo descritivo e exploratório com abordagem qualitativa, realizado com dez enfermeiras atuantes na Estratégia Saúde da Família (ESF) de um município do sudeste goiano, entre os meses de agosto e dezembro de 2015. O município conta com aproximadamente 98.737 habitantes e, no ano de 2015, possuía 11 equipes de (ESF) com cobertura de 42,16\% da população ${ }^{(8)}$. Os critérios de 
inclusão foram: enfermeiros que trabalhassem nas unidades de ESF por um período superior a 6 (seis) meses. Considerando os objetivos do estudo, não foram inseridos critérios de exclusão.

Para a coleta dos dados, utilizou-se a técnica de entrevista, com aplicação de um questionário semiestruturado, considerando o conhecimento das enfermeiras sobre as práticas integrativas e complementares e sua utilização na prática profissional. O convite para participação da pesquisa foi mediante ligação telefônica, seguida de agendamento prévio com os indivíduos que concordaram em participar.

As entrevistas, realizadas por pesquisadores devidamente capacitados, foram realizadas no local de trabalho das enfermeiras, em uma sala reservada, sendo gravadas e em seguida transcritas na íntegra.

Para tratamento dos dados, foi realizada análise de conteúdo temática proposta por Bardin. Segundo a autora ${ }^{(9)}$, na análise temática se buscará os núcleos de sentido que compõem a comunicação e cuja frequência teve sentido para o objeto analítico visado.

Para a teorização e análise dos dados, foi utilizada a Política Nacional de Práticas Integrativas Complementares (PNPIC) do Ministério da Saúde. Os aspectos éticos foram respeitados com parecer favorável do Comitê de Ética em Pesquisa da Universidade Federal de Goiás sob o n.ํㅜ 1.064.655/2015. Para preservar o anonimato, os participantes foram identificados por codinomes de flores típicas do cerrado: Turnera, Ipê, Polygala, Caliandra do cerrado, Juá, Lavoisiera, Orquídea, Flamboyant, Urtiga e Lobeira.

\section{RESUltados}

Foram entrevistadas 10 (dez) enfermeiras atuantes em 10 (dez) ESF do município, tendo em vista que 1 (uma) não aceitou participar do estudo.

No que se refere à caracterização das enfermeiras $100 \%$ eram do sexo feminino, sete $(70 \%)$ se declararam de cor parda, duas (20\%) se declararam como brancas e uma (10\%) se declarou negra. A média de idade foi de 32,7 anos e com 9,6 anos transcorridos da formação universitária. Quanto à religião, sete (70\%) relataram ser católicas, duas (20\%) evangélicas e uma (10\%) de outra confissão.

Dentre as perguntas realizadas através do instrumento de coleta de dados, cinco (50\%) das enfermeiras declararam saber o que são as PIC; 10 (100\%) admitiram a importância dessas práticas em, pelo menos, um aspecto relacionado com a saúde; 10 (100\%) disseram não conhecer uma ESF que ofereça essas práticas à população; cinco (50\%) relataram utilizar ou conhecer alguém que utilize as PIC; 10 (100\%) acreditam que as PIC trazem bons resultados aos pacientes; quatro (40\%) relataram que a comunidade possui bom conhecimento sobre as práticas; sete $(70 \%)$ relataram que a comunidade possui interesse relacionado com as PIC; oito (80\%) expressaram interesse em conhecer melhor estas práticas.

Segundo as enfermeiras, as PIC mais abrangentes, conhecidas e utilizadas pela comunidade e por elas na APS eram: acupuntura, fitoterapia, homeopatia, cromoterapia, shiatsu, automassagem (Do-In) e yoga, sendo a acupuntura a mais referida.

Ainda, diante das análises dos dados, foram estruturadas três categorias que versaram sobre o conhecimento e a percepção das enfermeiras sobre esta temática, que são:

\section{I - Conhecimento das enfermeiras sobre as PIC, dificuldade de conceituá-las e percepção como tratamento complementar}

Quando questionadas sobre o conceito e a denominação das PIC, percebeu-se que as enfermeiras apresentaram conhecimento superficial sobre as práticas, pois muitas não souberam defini-las, nominá -las e descrever seus benefícios:

Não conheço, por nome assim, não. (Turnera)

Mais ou menos, eu posso dizer que eu tenho um conhecimento superficial. (Polygala) 
Eu preciso de um esclarecimento maior, porque [após] muito tempo fora da faculdade, as coisas vão mudando bastante. (Lavoisiera)

Assim... Talvez a gente até já ouviu falar, mas não sabe o que é. (Flamboyant)

Assim... Eu não conheço muito bem; sei de alguma coisa que eu li, mas falar muito sobre isso eu não sei. (Lobeira)

Ainda sobre o conhecimento das enfermeiras no que se refere às PIC, encontrou-se que elas o têm sobre o uso dessas como uma técnica complementar aos tratamentos convencionais:

Essas práticas vão complementar o tratamento medicamentoso do paciente. (Caliandra do cerrado)

Sei que são práticas para complementar o tratamento do paciente, e também que servem para melhorar a qualidade de vida deles [...] Acredito que elas podem potencializar os resultados dos tratamentos. (Juá)

É questão de melhoria do tratamento, da adesão [...]; aliada ao tratamento correto, só vai trazer bons benefícios. (Urtiga)

Algumas enfermeiras relataram que apenas com o tratamento isolado pelas PIC não se obtém resultado, e ainda acrescentaram que alguns usuários se mostram resistentes em utilizar essas práticas apenas como um tratamento complementar, alguns chegando a abandonar o tratamento farmacológico convencional, como evidenciado na fala seguinte:

Só esse tipo de tratamento sozinho acho que não, porque ele ameniza e não cura [...] tem muitos que continuam com a medicação e tem outros que, por conta do resultado, querem parar de tomar o remédio, porque acham que só o chá resolve. (Flamboyant)

\section{II - Desafios e dificuldades na implementação das PIC na APS}

As participantes indicam desafios referentes à gestão pública para a implantação das PIC no SUS, mencionando recomendações técnicas feitas pelo Ministério da Saúde, com inviabilidade de implantação na realidade dos serviços:

Semana passada chegou uns materiais do Ministério falando sobre essas práticas, da fitoterapia, mas precisa de um espaço pra cultivar as plantas e tudo mais... Então, é uma coisa bem além daquilo que a gente tem disponível aqui. (Orquídea)

Algumas enfermeiras relataram que o conteúdo teórico sobre as PIC não foi abordado durante a graduação, fato este que dificulta a oferta de tais práticas na assistência prestada à comunidade:

[...]eu tenho formação há mais ou menos 10 anos; a grade curricular já mudou nesse tempo, então, assim: se hoje o conhecimento ainda é pouco, acho que naquela época era menor ainda. (Polygala)

Eu acredito que se elas [as $\mathrm{PIC}$ ] fossem abordadas como disciplina obrigatória, pré-requisito, igual às outras, e também como campo de estágio ou até mesmo na faculdade [...] eu acredito que seria de muita importância, suma importância. (Orquídea)

Eu não tive tanto contato durante a graduação [...] Se eu tivesse um acesso maior sobre o assunto, ia ser muito mais fácil de trabalhar com a comunidade, principalmente em ações de educação em saúde. (Lobeira)

Existem inúmeras dificuldades enfrentadas pelos profissionais de saúde quando se fala da implementação real das PIC aos usuários do SUS, como foi anteriormente mencionado. Porém, a sensibilização e a capacitação dos profissionais são assinaladas como de notória importância para uma eficaz disseminação desses conhecimentos, o que foi evidenciado pela fala a seguir:

Eu acho que é muito falha essa parte [...] Se nós déssemos um crédito maior pra isso e estudássemos e fôssemos mesmo na técnica de faculdade, talvez tivesse um valor maior e fosse visto com mais credibilidade. Eu acho que poderia funcionar melhor. (Lavoisiera) 
A predominância do modelo biomédico na formação e na assistência do cotidiano de trabalho também se mostra como um fator dificultante, conforme a fala a seguir:

Enquanto estudante a gente, até teve [abordagem] sobre esses tratamentos, mas como a gente tá acostumado só com a prática curativa, enquanto profissional de reabilitação, às vezes não usa tanto essas práticas, que poderiam ser utilizadas. (Orquídea)

\section{III - As PIC na APS como uma ferramenta de autocuidado e promoção da qualidade de vida}

Nesta categoria, quando questionadas sobre o desejo de implementação dessas práticas no SUS, as enfermeiras descrevem que isso seria muito importante, principalmente para melhorar a qualidade de vida da população, seja como uma forma de sensibilizá-la para a não-utilização de medicamentos sem a devida necessidade, seja como novas ferramentas de cuidado:

Assim... Elas já são incorporadas no SUS, mas aqui na cidade não. Porque eu acho que seria importante para o paciente, seria uma coisa a mais que a gente poderia oferecer pra ele pra melhorar a saúde, tanto física como mental. (Caliandra do cerrado)

[...] Elas auxiliariam e complementariam o tratamento dos pacientes do SUS. Além de melhorar a qualidade de vida deles também. (Juá)

Eu acho que melhora muito, porque as pessoas ficam viciadas em medicamentos, tem depressão, e talvez tendo um curso para elas sobre a importância dos chás, que ensinasse algumas coisas, melhoraria a qualidade de vida delas... (Ipê)

\section{DISCUSSÃO}

A partir dos resultados, é possível identificar que os enfermeiros possuíam pouco conhecimento sobre as PIC. Este pode ser considerado superficial pela própria formação de conceitos equivocados, o que pode gerar dificuldades na relação profissional-usuários ou com colegas praticantes dessas especialida$\operatorname{des}^{(10-11)}$.

Muitos profissionais deixam de buscar novas formas de satisfazer as necessidades dos clientes/usuários e realizar uma assistência integral devido à formação acadêmica curativista, influenciado pelo modelo biomédico, o que muitas vezes prioriza o desenvolvimento da tecnologia e a segmentação do cuidado, e não conhecem outras estratégias de saúde, como as PIC que valorizam o empoderamento do sujeito e formas mais naturais de tratamento ${ }^{(12)}$.

Como forma de enriquecer os recursos já existentes nos dias de hoje, torna-se necessário fortalecer as ações de promoção da saúde em relação às PIC com intuito de incluir outras modalidades terapêuticas de atenção à saúde, valorizando as políticas públicas e as necessidades especificas da comunidade ${ }^{(13)}$.

No estudo, as enfermeiras consideraram essas práticas complementares ao tratamento convencional, e ainda descreveram que as PIC mais abrangentes, conhecidas e utilizadas pela comunidade e por elas na APS eram: acupuntura, fitoterapia, homeopatia, cromoterapia, shiatsu, automassagem (Do- $I n)$ e yoga, sendo que a acupuntura foi referida por 100\% das entrevistadas. Todas essas práticas estão regulamentadas pela PNPIC e novas portarias do MS, como 849/2017 e a 145/2017 $7^{(4-5)}$.

O uso dessas práticas tem como intuito a complementação de outro tratamento ou a integração ao modelo convencional, seja como forma de redução de custos, incentivo as ações de prevenção de agravos e a manutenção da saúde por meio de tecnologias eficazes e segura que ressaltam a escuta acolhedora, a construção de vínculo terapêutico e a conexão sadia do indivíduo com o meio ambiente e a sociedade, o que está de acordo com a PNPIC (14-15). 
Profissionais que trabalham com estas práticas consideram o indivíduo como um todo (mente/corpo/ espírito), priorizando o foco na saúde e não na doença, e ainda buscam esclarecer aos indivíduos que a utilização destas ferramentas devem estar aliadas ao tratamento medicamentoso convencional, não substituindo o recurso farmacológico ${ }^{(14)}$, o que foi encontrado em muitas falas das enfermeiras.

Percebeu-se, ainda, nas falas das participantes que existem desafios para implementação das PIC na atenção primária, como falta de estrutura física e capacitação dos profissionais. Por mais valiosas e relevantes que sejam as técnicas envolvidas nas PIC há escassez de profissionais especializados, já que apenas alguns cursos de graduação na área da saúde ofertam em sua grade curricular disciplinas sobre o tema, o que limita o potencial daquelas práticas em contribuir com os serviços prestados à população usuária do $\operatorname{SUS}{ }^{(16)}$.

Disso decorre que o saber adquirido pela maioria dos acadêmicos é geralmente obtido por conhecimento empírico. No caso da Enfermagem, muitos estudantes também não conhecem os respaldos legais que o Conselho Federal de Enfermagem (COFEN) e a PNPIC oferecem aos profissionais desta classe para atuar com as $\mathrm{PIC}^{(17)}$.

Segundo estudos, a maioria dos profissionais de saúde acredita ser necessário a inclusão de matérias optativas ou obrigatórias relacionadas com as PIC durante a graduação, uma vez que elas possibilitariam aos acadêmicos o acesso a novas formas de assistência e cuidado. Contudo, só alguns profissionais acreditam que essas matérias deveriam ser ofertadas obrigatoriamente no curso $^{(18)}$.

A PNPIC traz como uma de suas diretrizes a importância de se ter profissionais qualificados para atuar na implementação dessas práticas na APS, juntamente com a comunidade, já que desse modo é possível o fortalecimento de vínculos terapêuticos e, consequentemente, maior adesão da comunidade a esses novos tratamentos ${ }^{(4)}$.

Os resultados deste estudo corroboram com a literatura ao descrever que as enfermeiras acreditam e relatam que as PIC proporcionam melhor qualidade de vida aos usuários e são importantes para promover a saúde. Estudos recentes têm evidenciado a efetividade da acupuntura e outras PIC no tratamento de disfunções fisiológicas como, por exemplo, disfunções temporomandibulares, doenças de cunho psicológico, osteoartrite, neuralgia do trigêmeo, doenças gástricas, enxaqueca, hipotonia muscular, hemiplegia e até mesmo a obesidade ${ }^{(18)}$.

Em um estudo realizado com 64 pessoas que possuíam dores crônicas e iniciaram um tratamento com quatro PIC (yoga, quiropraxia, acupuntura e massagem) em um período de três meses, relataram melhor bem-estar, aceitação e diminuição da dor, maior conscientização sobre a necessidade de um compromisso contínuo em seus próprios cuidados, autocontrole, e estavam mais motivados para buscar estratégias efetivas de enfrentamento ${ }^{(19)}$.

A incorporação das PIC na APS contribui significativamente para a humanização do cuidado e do atendimento. O cuidado humanizado resgata a autonomia dos indivíduos sobre a própria saúde, proporcionando estratégias para o autocuidado e para o cuidado da coletividade, uma vez que torna os usuários protagonistas do próprio processo de produção de saúde e da integralidade do atendimento. Com as PIC torna-se possível o diálogo entre todos esses elementos ${ }^{(15)}$, e também fortalecem o vínculo com os profissionais de saúde, como descrito por algumas enfermeiras do estudo, as quais relataram que a comunidade possui interesse em adquirir mais conhecimento sobre as PIC, o que pode fortalecer esta a relação profissional/usuário.

Pode-se perceber, em alguns contextos, que ainda há uma visão preconceituosa quanto às PIC, além do desconhecimento de suas contribuições no processo de cura de pacientes, podendo este ser um fato causador do desinteresse de diversos profissionais da saúde pelo uso de tais recursos ${ }^{(18)}$. Contudo, atualmente o MS, tem incentivado a capacitação e divulgação destas práticas na Atenção Primária à Saúde. Dados do segundo ciclo do Programa Nacional de Melhoria do Acesso e da Qualidade na Atenção Básica (PMAQ) em 2016 avaliaram mais de 30 mil equipes de atenção básica no território nacional e demonstrou que as 14 (quatorze) práticas incluídas na portaria n. 849/2017 do MS estão presentes nos serviços de saúde de todos o país ${ }^{(20)}$. 
Devido à baixa oferta de PIC na Atenção Primária do município estudado, nota-se a necessidade de multiplicar conhecimentos sobre as práticas com os profissionais de saúde, para assim, fortalecer a credibilidade em sua eficácia e também como forma de evidenciá-las e disseminá-las. Este estudo apresentou como limitação a realização da entrevista apenas com enfermeiros. Poderia obter-se o conhecimento e a percepção de gestores municipais e usuários dos serviços para estruturar programas e ações de saúde a partir da necessidade e singularidade da população.

\section{- CONCLUSÃO}

As enfermeiras participantes do estudo conheciam algumas PIC como a acupuntura, fitoterapia, yoga, cromoterapia, shiatsu e Do-In, sendo a acupuntura a mais mencionada. Também descreveram estas práticas como complementares ao tratamento convencional e que vários profissionais não conhecem muitas práticas e suas denominações, visto que não obtiveram este conhecimento na sua graduação e nem possuem atualizações sobre o tema. Também não dispõem de estrutura física e recursos humanos adequados para implementação das mesmas em seus locais de trabalho.

Devido a isso, percebe-se o quanto os enfermeiros carecem de informações sobre as PIC, sua utilização, seus benefícios e sobre as regulamentações do Conselho Federal de Enfermagem e do MS, que autorizam e incentivam a utilização das mesmas na APS e em outros níveis de atenção, principalmente no que tange à promoção da saúde e prevenção de doenças.

Pôde-se verificar que uma das estratégias para modificar esse cenário seria a reestruturação dos componentes curriculares dos cursos da área da saúde, principalmente dos cursos de Enfermagem, inserindo disciplinas e/ou estágios na área das PIC, para que cada vez mais os profissionais estejam capacitados para atuar com tais recursos nos cenários de assistência. Além disso, seria desejável a inclusão das PIC nas temáticas abordadas no contexto da educação permanente em saúde, para atualizar os conhecimentos dos profissionais, ao viabilizar formações e capacitações técnicas para o atendimento.

\section{REFERÊNCIAS}

1. Melo SCC, de Santana RG, Santos DC, Alvim NAT. Práticas complementares de saúde e os desafios de sua aplicabilidade no hospital: visão de enfermeiros. Rev. bras. enferm. [Internet] 2013;66(6) [acesso em 16 nov 2016]. Disponível: http://dx.doi.org/10.1590/S0034-71672013000600005.

2. Gnatta JR, Kurebayashi LFS, Turrini RNT, da Silva MJP. Aromaterapia e enfermagem: concepção histórico-teórica. Rev. esc. enferm. USP. [Internet] 2016;50(1) [acesso em 17 mar 2017]. Disponível: http://dx.doi.org/10.1590/S0080623420160000100017.

3. Silva MJP. Práticas alternativas e complementares e enfermagem. Grupo de práticas alternativas ou complementares em saúde. 2014. [Internet] [acesso em: 21 out 2015]. Disponível: http://www.ee.usp.br/site/index.php/paginas/mos$\operatorname{trar} / 752 / 1343 / 107$.

4. Ministério da Saúde (BR). Secretaria de Atenção à Saúde. Departamento de Atenção Básica. Política Nacional de Práticas Integrativas e Complementares no SUS (Atitude de Ampliação de Acesso). [Internet] Brasília: Ministério da Saúde; 2014 [acesso em 19 mar 2016]. Disponível: http://bvsms.saude.gov.br/bvs/publicacoes/politica_nacional_praticas_integrativas_complementares_2ed.pdf.

5. Ministério da Saúde (BR). Portaria n. 849, de 27 de março de 2017. Inclui a Arteterapia, Ayurveda, Biodança, Dança Circular, Meditação, Musicoterapia, Naturopatia, Osteopatia, Quiropraxia, Reflexoterapia, Reiki, Shantala, Terapia Comunitária Integrativa e Yoga à Política Nacional de Práticas Integrativas e Complementares. Diário Oficial União, 28 mar 2017; Seção 1. 
6. Fischborn AF, Machado J, Fagundes NC, Pereira NM. A Política das práticas integrativas e complementares do SUS: o relato de experiência sobre a implementação em uma unidade de ensino e serviço de saúde. Cinergis. [Internet] 2016;17(4) [acesso em 26 mai 2017]. Disponível: https: http://dx.doi.org/10.17058/cinergis.v17i0.8149.

7. George JB, colaboradores. Teorias de enfermagem: os fundamentos à prática profissional. $4^{\mathrm{a}}$ ed. Porto Alegre: Artmed; 2000.

8. Ministério da Saúde (BR). Departamento de Atenção Básica-DAB. Histórico de cobertura de saúde da família. [Internet] 2017 [acesso em 16 mar 2017]. Disponível: https://egestorab.saude.gov.br/paginas/acessoPublico/relatorios/relHistoricoCoberturaAB.xhtml.

9. Bardin L. Análise de conteúdo. São Paulo: Edições 70; 2011.

10. Santos MC, Tesser CD. Um método para a implantação e promoção de acesso às práticas integrativas e complementares na atenção primária à saúde. Ciênc. saúde coletiva. [Internet] 2012;17(11) [acesso em 26 nov 2016]. Disponível: http://dx.doi.org/10.1590/S1413-81232012001100018.

11. Thiago SCS, Tesser CD. Percepção de médicos e enfermeiros da estratégia de saúde da família sobre terapias complementares. Rev. Saúde Pública. [Internet] 2011;45(2) [acesso em 16 nov 2016]. Disponível: http://dx.doi.org/10.1590/ S0034-89102011005000002.

12. Bruning MCR, Mosegui GBG, Vianna CMM. A utilização da fitoterapia e de plantas medicinais em unidades básicas de saúde nos municípios de Cascavel e Foz do Iguaçu - Paraná: a visão dos profissionais de saúde. Ciênc. saúde coletiva. [Internet] 2012;17(10) [acesso em 04 fev 2017]. Disponível: http://dx.doi.org/10.1590/S1413-81232012001000017.

13. Telesi Junior E. Práticas integrativas e complementares em saúde, uma nova eficácia para o SUS. Estud. av. [Internet] 2016;30(86) [acesso em 11 mar 2017]. Disponível: http://dx.doi.org/10.1590/S0103-40142016.00100007.

14. Schveitzer MC, Esper MV, da Silva MJP. Práticas integrativas e complementares na atenção primária em saúde: em busca da humanização do cuidado. Mundo da saúde. [Internet] 2012;36(3) [acesso em 29 mai 2017]. Disponível: http:// www.saocamilo-sp.br/pdf/mundo_saude/95/6.pdf.

15. Ischkanian PC, Pelicioni MCF. Desafios das práticas integrativas e complementares no SUS visando a promoção da saúde. Journal of Human Growth and Development. [Internet] 2012;22(2) [acesso em 02 fev 2017]. Disponível: http:// dx.doi.org/10.7322/jhgd.44936.

16. Lima KMSV, Silva KL, Tesser CD. Práticas integrativas e complementares e relação com promoção da saúde: experiência de um serviço municipal de saúde. Interface (Botucatu). [Internet] 2014;18(49) [acesso em 10 jun 2017]. Disponível: http://dx.doi.org/10.1590/1807-57622013.0133.

17. Gavin ROS, de Oliveira MHP, Gherardi-Donato ECS. Terapias alternativas complementares: uma visão do conhecimento dos acadêmicos de enfermagem. Cienc. Cuid. Saude. [Internet] 2010;9(4) [acesso em 27 abr 2017]. Disponível: http://dx.doi.org/10.4025/cienccuidsaude.v9i4.13827.

18. Gontijo MBA, Nunes MF. Práticas integrativas e complementares: conhecimento e credibilidade de profissionais do serviço público de saúde. Trab. educ. saúde. [Internet] 2017;15(1) [acesso em 26 mai 2017]. Disponível: http://dx.doi. org/10.1590/1981-7746-sol00040.

19. Eaves ER, Sherman KJ, Ritenbaugh C, Hsu C, Nichter M, Turner JA, et al. A qualitative study of changes in expectations over time among patients with chronic low back pain seeking four CAM therapies. Complementary and Alternative Medicine. [Internet] 2015;15 [acesso em 29 jan 2018]. Disponível: https://doi.org/10.1186/s12906-015-0531-9.

20. Ministério da saúde (BR). Secretaria de Atenção à Saúde. Departamento de Atenção Básica. PNPIC é ampliada. [Internet] Brasília: Ministério da Saúde; 2017 [acesso em 29 jan 2018]. Disponível: http://dab.saude.gov.br/portaldab/ noticias.php?conteudo=_\&cod=2331. 